\title{
REDUCED GRAPHENE OXIDE SENSOR FOR QUANTIFICATION OF METABOLITES IN EXHALED BREATH CONDENSATE
}

\author{
Azam Gholizadeh, Damien Voiry, Clifford Wiesel, Andrew Gow, Robert Laumbach, \\ Howard Kipen, Manish Chhowalla, and Mehdi Javanmard \\ Rutgers University, USA
}

\begin{abstract}
This work reports nitrite detection in EBC samples using reduced graphene oxide as the sensing material. Nitrite content in EBC can be a potential biomarker for inflammation in the respiratory system. The performance of the sensor was characterized in standard nitrite solutions using cyclic voltammetry and square wave voltammetry and then was applied to patient EBC samples. The sensor has a sensitivity of $0.21 \mu \mathrm{A} \mu \mathrm{M}^{-1} \mathrm{~cm}^{-2}$ in the range of $20-100 \mu \mathrm{M}$ and $0.1 \mu \mathrm{A} \mu \mathrm{M}^{-1}$ $\mathrm{cm}^{-2}$ in 100-1000 $\mu \mathrm{M}$ nitrite concentration with a low detection limit of $830 \mathrm{nM}$ in EBC matrix.
\end{abstract}

\section{INTRODUCTION}

Exhaled breath condensate (EBC) has been widely studied as a noninvasive source of biomarkers in chronic respiratory diseases. Although EBC has great potential as a source of biomarkers in many chronic respiratory conditions such as asthma, the low samples volume and concentrations of biomarkers within EBC present analytical challenges.

Exhaled breath contains gaseous materials such as nitric oxide and carbon monoxide, and EBC contains non-volatile compounds including small molecules such as water, hydrogen peroxide, nitrite and nitrate, and larger molecules such as eicosanoids, proteins, and even DNA [1]. Among these different substances, in this work we are interested in detection of nitrite content. The main source of nitrite in the inner lining of the airway is nitric oxide (NO) that is produced from L-arginine. In aqueous solution NO reacts rapidly with reactive oxygen species to form more stable form of nitrogen oxide such as nitrite $\left(\mathrm{NO}_{2}\right)$ and nitrate $\left(\mathrm{NO}_{3}\right)$ [2]. Increased level of $\mathrm{NO}$ has shown correlation to inflammatory conditions such as asthma and cystic fibrosis [3]. The increased level of NO in asthma has been suggested to be due to an increased expression of inducible NO synthase (iNOS) in the bronchial epithelium [4].

Common methods for detection nitrite are the spectrophotometric assay (Griess reaction), which is a fluorimetric method, chemiluminescence or ion chromatography [5]. The detection limit of the fluorimetric method is $0.1 \mu \mathrm{M}$. The Griess method, however, has a higher detection limit compared to the chemiluminescence method, which has a lower detection limit in the $\mathrm{nM}$ range. Nitrite content detected in EBC is in the $\mu \mathrm{M}$ range, which is compatible with these methods detection limits.

However, inspite of the low detection limit of these methods, often they require pretreatment steps on EBC samples to enable a suitable reaction or eliminate interfering compounds such as chlorine [6]. Usually electrochemical methods do not need this type of pretreatment and easily can be miniaturized to be used for point-of-care diagnostics and can potentially detect nitrite at a specific redox potential in real-time without interference from other compounds in the EBC matrix. However they do not have a detection limit as low as the optical techniques and require milliliters of sample volume.

Electrochemical detection of nitrite is based on either oxidation or reduction of the nitrite [7]. Oxidation of nitrite is usually preferred with the final product of $\mathrm{NO}_{3}$ since main limitation of interference sources such as oxygen in reduction can be avoided. However, the problem of oxidation is its high over-potential [8] thus many attempts have been made to develop novel electrode materials in recent years [9]. Among of them, graphene based electrodes have been widely used due to small residue current, wide potential window, excellent chemical stability and easy functionalization. In this work we used graphene based electrodes for detection of nitrite. We fabricated a nitrite sensor using a combination of benefits offered by screen-printed electrodes and electrochemically reduced Graphene Oxide with low detection limit. To the best of our knowledge, this is first electrochemical sensor that directly measures nitrite in EBC samples with this level of sensitivity.

Another issue common in many graphene based electrochemical sensors, is that they usually use simple drop cast methods to deposit GO on top of the electrode which can result in very different results from sample to sample and has an agglomeration problem. Here we used a modified drop cast method to have a thinner layer on the electrode surface. Also by using the PDMS membrane as the electrochemical cell, voltammetric measurement of low volume EBC samples (100 $\mu 1)$ can be easily done.

\section{EXPERIMENTAL}

To calibrate the sensor response we tested sodium nitrite spiked into acetate and phosphate buffer. Graphene oxide was prepared using hummers method. The morphology of graphene oxide was characterized using field emission scanning microscopy (SEM) with Shimadzu SS 550 SEM instrument. AFM was performed using Digital Instruments Nanoscope IV in tapping mode with standard cantilevers with spring constant of $40 \mathrm{~N} \mathrm{~m}^{-1}$ and tip curvature $<10 \mathrm{~nm}$. FT-Raman spectra were recorded using Horiba Johin-Yvon Micro Raman Spectrometer, equipped with a microscope having a $532 \mathrm{~nm}$ laser as the source of its excitation beam. Electrochemical measurements were performed with PSTAT Princeton instrument in ambient condition. All potentials are with respect to $\mathrm{Ag} / \mathrm{AgCl}$ reference electrode.

The steps of modification of the gold electrode with rGO are shown in figure 1 . First, a $3 \mu \mathrm{L}$ aliquot of graphene oxide suspension that was synthesized from graphite powder by hummers method was dropped on the gold electrode surface. Then thin glass slide was placed on top of droplet and extra solution was removed and surface was dried under room temperature. Then GO thin layer was reduced electrochemically in acetate buffer with a $\mathrm{pH}$ of 5.5 using cyclic voltammetry between -1.6 to $0 \mathrm{~V}$ with scan rate of $25 \mathrm{mV}$ for 30 cycle under continuous $\mathrm{N}_{2}$ purging.

For fabrication of an electrochemical cell with microliters of 
volume, a thin layer of PDMS was applied on top of the SPE electrode holder and then the top PDMS well containing a $8 \mathrm{~mm}$ hole was bonded onto it using $\mathrm{O}_{2}$ plasma. During $\mathrm{O}_{2}$ plasma treatment, the rGO layer and the connection pads were protected with glass slide. a)

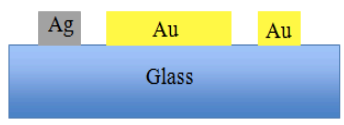

c)

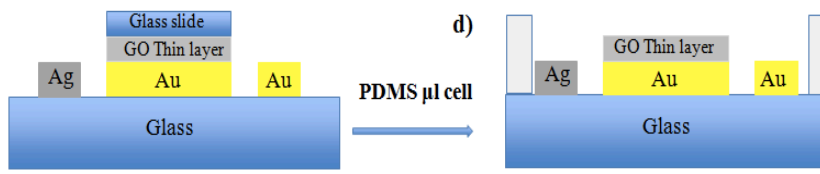

e)

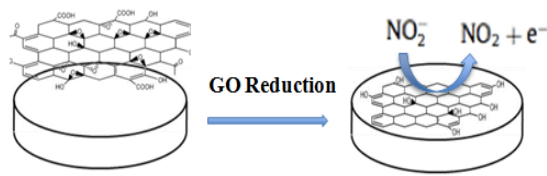

b)

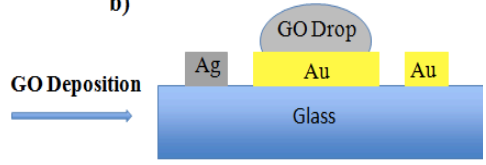

d)
Figure 1: Schematic of nitrite sensor fabrication steps: a) screen print electrode with working and counter electrode from gold, reference electrode from $\mathrm{AgAgCl}$ past. b) drop cast solution contains GO. c) Using glass slide on top of drop to have very thin layer of GO. d) Fabrication PDMS electrochemical cell for low volume EBC samples. e) Electrochemically reduction of $G O$.

\section{RESULTS AND DESCUSSIONS}

\section{Characterization of Modified Electrodes}

Characterization of GO morphology has been done with AFM and SEM. The level of reduction of GO also has been quantified with Raman spectroscopy. Figure 2a shows AFM images of GO on $\mathrm{Si} / \mathrm{SiO}_{2}$ substrate. In most areas there is a uniform flat GO layer with wrinkles and some agglomerated area that can be produce during vaporization of solvent which perhaps can be avoided using lower concentration of GO solution or drying in vacuum, however because we are interested in the electrochemical properties instead of the absolute electrical properties of GO, and because the electrochemical edges of GO are more sensitive than the flat layer, we intentionally did not try to remove these defects. However, this can be further studied using impedance spectroscopy to learn more about the effect of these defects on electrochemical behavior of the rGO layer.

Also an SEM image was taken from a larger area from the GO layer directly deposited on gold electrode (figure $2 b$ ). It shows that even in the gold working electrode with a roughness of several microns we have a covered uniform layer in most of the area. In comparison to common drop cast methods that usually are used in electrochemically-modified electrodes this method can give a much larger area of a few GO layers covered without agglomeration.

The efficiency of electrochemical reduction of GO also was investigated using raman spectroscopy. Representative data shows the average of three measurements in different areas of each sample. Figure $2 \mathrm{C}$ is the raman spectrum of $\mathrm{GO}$ layer before reduction and figure $2 \mathrm{D}$ shows the raman spectrum of rGO after 30 cycles of reduction.
In carbon-based materials, the main features in the Raman spectra are the $G$ and $D$ peaks. These peaks arise from vibration of $\mathrm{sp}^{2}$ carbon, appearing around 1580 and $1350 \mathrm{~cm}^{-1}$ respectively. The overtone of the $\mathrm{D}$ peak appeared around 2700 $\mathrm{cm}^{-1}$ is called 2D peak. Unlike mechanically exfoliated graphene, because GO is more disordered, thus its 2D band usually has low intensity. The peaks that can be used for their ability to distinguish between GO and rGO are G and D peaks and their ratio. Also the $\mathrm{G}$ peak of $\mathrm{GO}$ and $\mathrm{rGO}$ with respect to graphene and graphite gets shifted into higher frequencies (1598 $\mathrm{cm}^{-1}$ ) because of defects. Even in the case of thermal reduction of GO usually $\mathrm{I}_{\mathrm{D}} / \mathrm{I}_{\mathrm{G}}$ remains constant, the increased $\mathrm{I}_{\mathrm{D}} / \mathrm{I}_{\mathrm{G}}$ ratio of rGO after electrochemical reduction has been reported in theliterature. In this study, this ratio exhibited a significant increase compare to GO (from 0.87 to 1.1). This shows restoration of $\mathrm{sp}^{2}$ carbon and a decrease in the average size of $\mathrm{sp}^{2}$ domains after electrochemical reduction of GO. An increase in the $2 \mathrm{D}$ peaks also suggests better graphitization [10]. a)

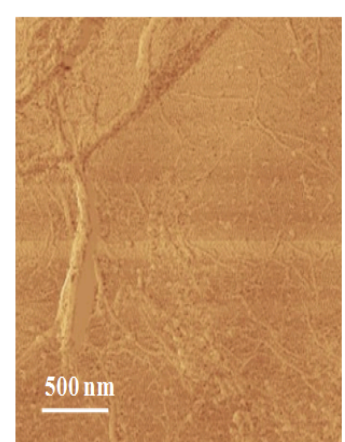

c)

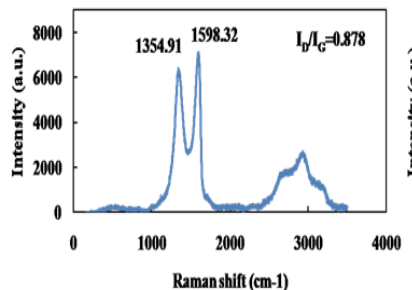

b)

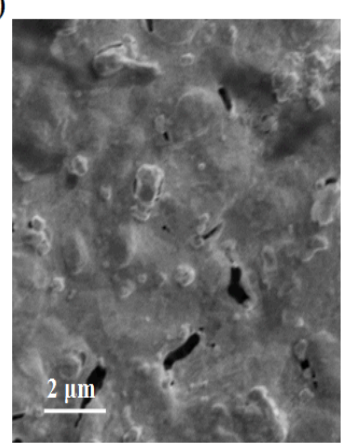

d)

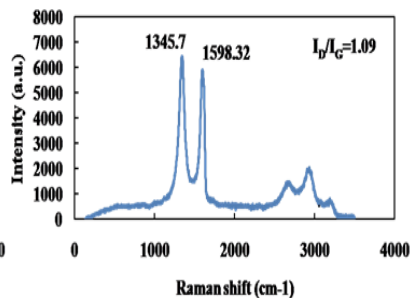

Figure 2. a) $A F M$, b) SEM of $G O$ thin layer. c) Raman of $G O$, d) Raman of $r G O$.

Electrochemical response toward nitrite

After we achieved properly reduced GO, we get through electrochemical performance of layer toward our analyte of interest, nitrite. As our final goal is fabrication of a miniaturized sensor that can be operate in ambient conditions, so even $\mathrm{O}_{2}$ can react with analyte, we preferred to do all our experiment in presence of $\mathrm{O}_{2}$ to understand how the sensor works in ambient conditions. As seen in the results of the EBC samples, the voltage of the oxidation peaks gets shifted to positive voltages, it is better to work in a lower over-potential thus acetate buffer with $\mathrm{pH} 6$ was selected as electrolyte. Also $\mathrm{pH}$ in EBC samples of patients with inflammatory disease has been reported as acidic [11]. Thus $\mathrm{pH}$ of 6 is more similar to the $\mathrm{pH}$ of EBC samples obtained from asthma patients. Also the performance of rGO modified electrodes compare to SPE and GO deposited electrode was investigated. Figures $3 \mathrm{~A}$ and $3 \mathrm{~B}$ shows the response of GO and $\mathrm{rGO}$ modified electrodes in presence of 100 
to $1000 \mu \mathrm{M}$ nitrite respectively in $25 \mathrm{mV} / \mathrm{s}$ scan rate.

The performance of the fabricated electrodes was confirmed in standard electrolytes containing different concentrations of nitrite. This motivated us to test the electrode in EBC to understand the effect of the complex biological matrix, which was necessary before trying to measure basal levels of nitrite content in EBC.

We fabricated an electrochemical sensor capable of detecting nitrite content in real EBC samples in the $\mu \mathrm{M}$ range. Cyclic Voltammetry and Square Wave Voltammetry were used to measure the current from different concentrations of nitrite solution in buffer spiked into EBC. Also, the analytical performance of the nitrite sensor was explored using square wave voltammetry (SWV). SWV measurements were conducted from 0 to $0.9 \mathrm{~V}$. Figures $5 \mathrm{~A}$ and $5 \mathrm{~B}$ display the square wave voltammograms of nitrite in the range of $(2-1000 \mu \mathrm{M})$. The peak current in around $0.7 \mathrm{~V}$ depends on nitrite concentration. Figure 5C and 5D shows calibration curve based on SWV method.

a)

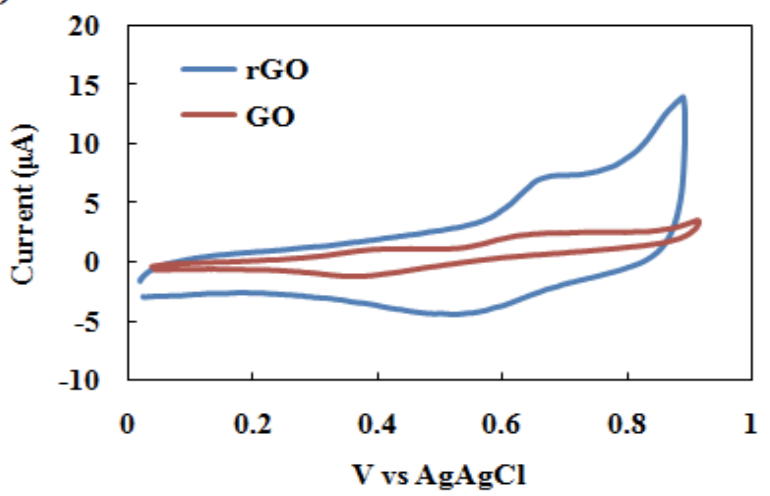

b)

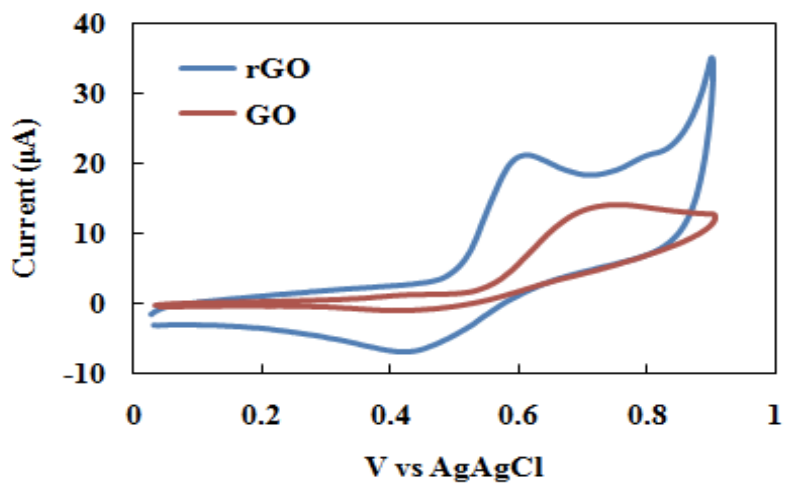

Figure 3. Cyclic voltammetry of $G O$ and $r G O$ in a) buffer solution contains $100 \mu \mathrm{M}$ and b) $1 \mathrm{mM}$ nitrite.

Seven different EBC samples were tested to determine the accuracy of the sensors in detecting nitrite for different EBC samples. SWV was measured for each of the samples separately (figure 5a). The slight difference between the oxidation potential may be because of the different complex matrix of EBC from patient to patient. Based on their oxidation current; their concentrations were compared to data that was collected with ozone based chemiluminescence [13]. Figure 6B shows the calibration curve based of current respect predicted concentration using chemiluminescence method. As can be seen there is a linear relationship between them.

\section{CONCLUSION}

In this study the enzyme-free nitrite sensor was fabricated using the unique properties of Graphene combined with SPEs. The use of the PDMS membrane allows us to measure low volumes of EBC samples. After optimization of various parameters in purified buffer solution, we tested real EBC samples. We obtained the calibration curve using various spiked nitrite concentrations in EBC. The sensitivity of the sensor is $0.21 \mu \mathrm{A}$ $\mu \mathrm{M}^{-1} \mathrm{~cm}^{-2}$ in the range of $20-100 \mu \mathrm{M}$ and $0.1 \mu \mathrm{A} \mu \mathrm{M}^{-1} \mathrm{~cm}^{-2}$ in $100-1000 \mu \mathrm{M}$ with detection limit of $830 \mathrm{nM}$. The data for a small number (7) of real EBC samples was also collected and compared with chemiluminescence methods. The sensor was demonstrated to measure nitrite content with high accuracy in the $\mu \mathrm{M}$ range of concentration which for most cases is the typical range of nitrite in EBC samples. Detection was even demonstrated in the nanoMolar range, however enabling robust operation requires further improvement. This can be done either through modification of the geometry of the sensor or through use of graphene-nanoparticle composites. a)
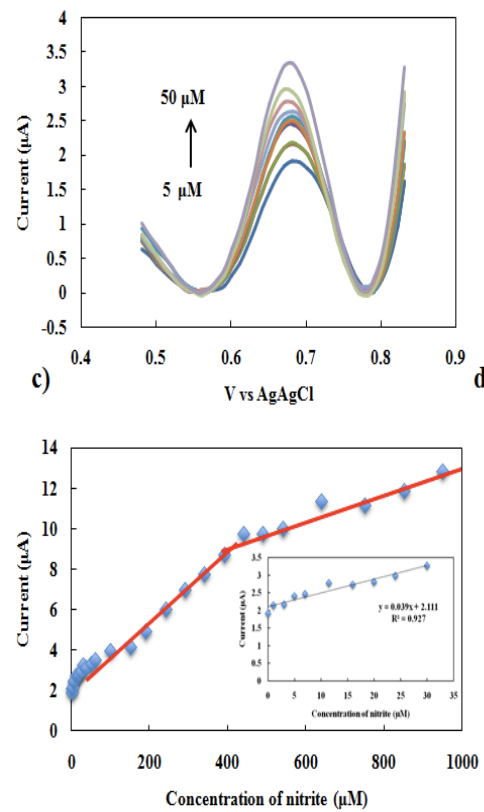

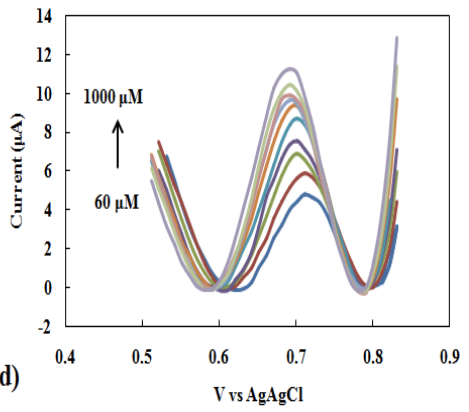

Figure 4. a,b) SWV of different concentrations of nitrite spiked in EBC sample. $c$,d) calibration curves. Inset of $c$ is calibration curve for lower concentration range. 
a)

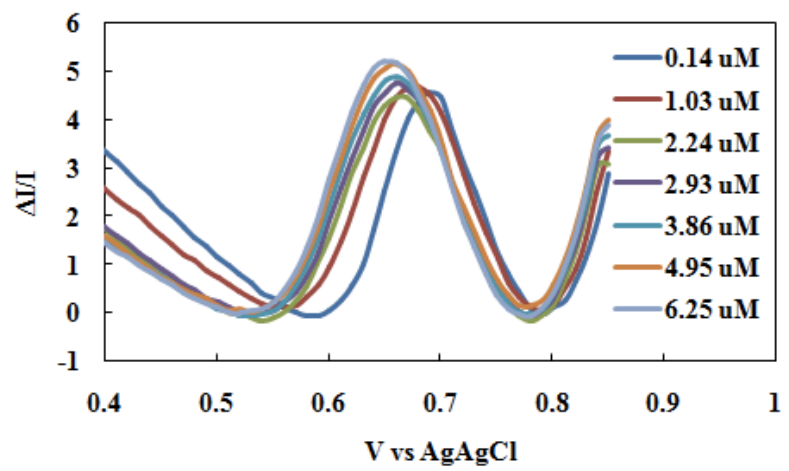

b)

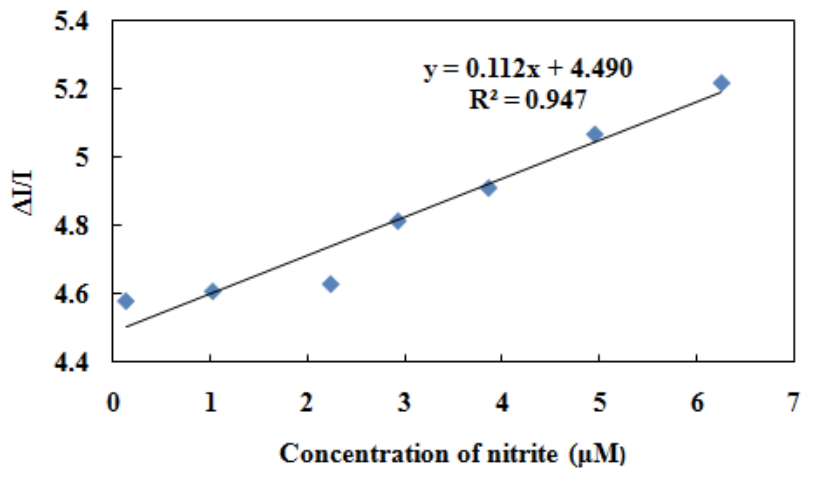

Figure 5. a) SWV of seven different EBC samples, b) calibration curve using photoluminescence nitrite concentration data.

\section{References:}

[1]. Mutlu, G. M.; Garey, K. W.; Robbins, R. A.; Danziger, L. H.; Rubinstein, I., Collection and analysis of exhaled breath condensate in humans. Am J Respir Crit Care Med 2001, 164 (5), 731-7.

[2]. Gaston, B.; Drazen, J. M.; Loscalzo, J.; Stamler, J. S., The biology of nitrogen oxides in the airways. Am J Respir Crit Care Med 1994, 149 (2 Pt 1), 538-51.

[3]. Francoeur, C.; Denis, M., Nitric oxide and interleukin8 as inflammatory components of cystic fibrosis. Inflammation 1995, 19 (5), 587-98.

[4]. Hamid, Q.; Springall, D. R.; Riveros-Moreno, V.; Chanez, P.; Howarth, P.; Redington, A.; Bousquet, J.; Godard, P.; Holgate, S.; Polak, J. M., Induction of nitric oxide synthase in asthma. Lancet 1993, 342 (8886-8887), 1510-3.

[5]. Balint, B.; Kharitonov, S. A.; Hanazawa, T.; Donnelly, L. E.; Shah, P. L.; Hodson, M. E.; Barnes, P. J., Increased nitrotyrosine in exhaled breath condensate in cystic fibrosis. Eur Respir J 2001, 17 (6), 1201-7.

[6]. Adarsh, N.; Shanmugasundaram, M.; Ramaiah, D., Efficient reaction based colorimetric probe for sensitive detection, quantification, and on-site analysis of nitrite ions in natural water resources. Anal Chem 2013, 85 (21), 10008-12.

[7]. Wu, H.; Fan, S.; Zhu, W.; Dai, Z.; Zou, X., Investigation of electrocatalytic pathway for hemoglobin toward nitric oxide by electrochemical approach based on protein controllable unfolding and in-situ reaction. Biosens Bioelectron 2013, 41, 589-94.
[8]. Wang, S.; Yin, Y.; Lin, X., Cooperative effect of Pt nanoparticles and $\mathrm{Fe}$ (III) in the electrocatalytic oxidation of nitrite. Electrochemistry communications 2004, 6 (3), 259-262.

[9]. Davis, J.; Compton, R. G., Sonoelectrochemically enhanced nitrite detection. Analytica Chimica Acta 2000, 404 (2), 241-247.

[10]. Yue, R.; Lu, Q.; Zhou, Y., A novel nitrite biosensor based on single-layer graphene nanoplatelet-protein composite film. Biosensors and Bioelectronics 2011, 26 (11), 4436-4441.

[11]. Kostikas, K.; Papatheodorou, G.; Ganas, K.; Psathakis, K.; Panagou, P.; Loukides, S., pH in expired breath condensate of patients with inflammatory airway diseases. American Journal of Respiratory and Critical Care Medicine 2002, 165 (10), 1364-1370.

[12]. Tung, V. C.; Allen, M. J.; Yang, Y.; Kaner, R. B., High-throughput solution processing of large-scale graphene. Nature nanotechnology 2009, 4 (1), 25-29.

[13]. Pettit, A. P.; Kipen, H.; Laumbach, R.; OhmanStrickland, P.; Kelly-McNeill, K.; Cepeda, C.; Fan, Z.-H.; Amorosa, L.; Lubitz, S.; Schneider, S., Disrupted Nitric Oxide Metabolism from Type II Diabetes and Acute Exposure to Particulate Air Pollution. PloS one 2015, 10 (12). 